\title{
Fast B-Mode Ultrasound Image Simulation of Deformed Tissue
}

\author{
Orcun Goksel and Septimiu E. Salcudean \\ Department of Electrical and Computer Engineering, University of British Columbia, Vancouver, BC, Canada
}

\begin{abstract}
This paper presents a fast image synthesis procedure inside elastic volumes under deformation simulated by the finite element method (FEM). Given the node displacements of a mesh and the 3D image voxel data of a volume prior to deformation, the method maps the image pixels, to be synthesized, from the deformed configuration back to the nominal pre-deformed configuration, where the pixel intensities are obtained easily through interpolation in the regular-grid structure of the voxel volume. This mapping requires the identification of the mesh element enclosing each image pixel, in order to use its corresponding shape function for smooth interpolation. To accelerate this point location operation, a fast method of marking the projection of the deformed mesh on the image pixels at every frame is introduced.

In order to evaluate our method, a deformable tissue phantom was constructed and its 3D ultrasound volume was acquired in its nominal state. B-mode images of the phantom were then synthesized under the simulated deformation of an ultrasound probe. Results show that realistic B-mode images can be simulated in real-time with the proposed technique, even under large deformations. The technique is also implemented on a real-time system for ultrasound exploration with deformation.
\end{abstract}

\section{INTRODUCTION}

Medical simulators involving real-time imaging modalities, such as ultrasound, necessitate rapid and realistic image rendering of deformed tissue in response to probe or tool manipulation by a trainee. For real-time performance, tissue deformation, typically modeled by the finite element method (FEM), must be computed on a mesh having much coarser elements than the typical resolution of medical imaging modalities. The deformation is predicted in the form of node displacements of this mesh. Our work addresses the computational problems related to rapid image generation in a deformed 3D mesh. The need for real-time image synthesis during the simulation of ultrasound makes it our target application. Nevertheless, the techniques presented in this paper are also applicable to other imaging modalities that need to slice deformed meshes in real-time.

Image generation inside deformed tissue has some concepts in common with the elastic registration [1], [2] and the ultrasound volume reconstruction [3], [4] literature, where reconstruction refers to generating $3 \mathrm{D}$ voxel volume data from individual scans. However, compared to these fields that generally process images offline, real-time medical image generation presents additional computational challenges, which have been studied for ultrasound B-mode image simulation in the literature. There exist two approaches for ultrasound image synthesis, the generative approach and the interpolative approach. The former models the ultrasonic wave propagation by using accurate representations of the probe, the tissue scatterers, and the wave interaction [5]. This complex and time-consuming approach is not suitable for real-time applications. The latter approach slices images from a pre-existing voxel volume data. UltraSim [6], which is one of the first commercial ultrasound image simulators, and several others [7] follow this latter approach.

Existing ultrasound simulators do not allow for tissue deformation. However, in many medical procedures such as prostate brachytherapy, brain surgery, or breast biopsy, significant deformation is caused by medical tools or by the ultrasound probe. In certain applications, such as diagnosis of deep-vein thrombosis (DVT), deformation observed in ultrasound images during deliberate probe indentation contains essential diagnosis information. Fast synthesis of ultrasound images in soft tissues under deformation will facilitate the development of training simulators. With this goal, a DVT diagnosis simulator was proposed in [8], [9]. It simulates the probe pressure by first slicing an image from the 3D ultrasound data set and then applying a 2D elastic deformation using quadtree-splines to this image. This 2D deformation applied is pre-computed offline by registering segmented anatomy from pre-deformed to deformed 3D models. Real-time ultrasound image slicing using physicallyvalid 3D deformation models has not been addressed in the literature. Our work is motivated by this need for real-time realistic B-mode ultrasound simulation in medical training environments involving tissue deformation.

The paper is organized as follows. First, the interpolation approach of mapping image pixels back to the predeformed volume is outlined. Then, the problems related to this approach, specifically the pixel location problem, and our proposed numerical treatments to accelerate this scheme are presented. The proposed technique is next applied to real-time ultrasound synthesis in tissue deformed by the ultrasound probe. The results are demonstrated on a tissuemimicking ultrasound phantom. A discussion of the limitations and possible future extensions conclude this paper.

\section{METHODS}

Our goal is to generate planar image slices inside a volume under deformation. We assume that the 3D image data set of the entire volume is available to us a priori. The ultrasound reconstruction literature extensively addresses generating these $3 \mathrm{D}$ volume data.

\section{A. Inverting the deformation}

For each image frame to be generated in real-time, 3D locations of image pixels are known from the probe po- 
sition/orientation since pixels of an image lie on a planar regular-grid structure. In order to synthesize an image, the intensity values (gray-values) at these known locations are to be interpolated given the reconstructed voxel data. However, due to deformation there exists a different local transformation for each voxel from the nominal predeformed configuration to the later deformed configuration. This disturbs the regular-grid structure of the voxel data in the deformed state in 2D. Since interpolating the image pixel locations in this scattered given data would be complex and time-consuming, in this paper we propose the approach of transforming (undeforming) the image back into the nominal frame where the given voxels lie on a regular grid structure.

\section{B. Image Pixels in an FEM Tessellation}

Deformation is commonly simulated by the FEM, in which tessellations are typically much coarser than medical imaging resolutions due to computational constraints. In the FEM, deformation is defined in the form of node displacements of an overlaid mesh. For example, consider the situation in Fig. 1(a)(top) where an image plane slices an object deformed under the constraints shown with the arrows as simulated. The undeformed image pixel positions, at which the intensities are to be interpolated, can then be computed using the shape functions of the elements enclosing these pixels (see Fig. 1(a)(bottom)). The overall interpolation process becomes as follows:
For each image pixel $P$
1. Find the element $e_{i}$ enclosing $P$
2. Compute the transformation $T$ for $e_{i}$ using its
deformed-to-nominal node displacements
3. Find the undeformed pixel position $T P$
4. Interpolate $T P$ in the given voxel data

Steps 2 to 4 are constant-time operations. On the other hand, Step 1 is the bottleneck of this approach. It is called the point location problem in computational geometry and has been extensively studied in the literature resulting in common techniques such as slab decomposition and trapezoidal maps. However, any such method unfortunately would not allow real-time processing of conventional image resolutions. Indeed, the point location routine in the Quickhull package [10] locates the pixels of a single typical image presented in our Results section, in over 30 seconds. The rest of this paper focuses on accelerating this first step.

\section{Exploiting the Image Geometry for Fast Point Location}

Note that the enclosing element of an image pixel depends on both mesh deformation and the image position/orientation; and hence, it cannot be computed offline. Nevertheless, one can exploit the fact that image pixels are at locations that are (i) ordered and (ii) regularly-spaced in the imaging plane, rather than being scattered points. The former allows us to locate any pixel by only knowing where the mesh intersects the image. For instance, in the 2D example in Fig. 1(b), assume that only the pixels near the element borders, marked with stars, are located and



(a)

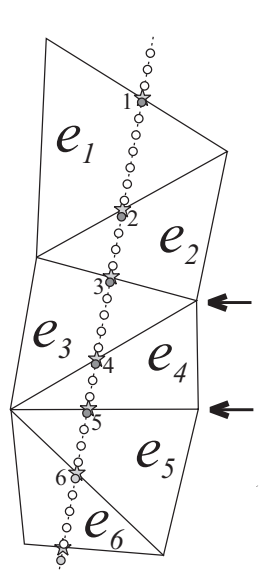

(b)



(c)
Fig. 1. (a) A deformed 2D tissue mesh under constraints shown by arrows and a cutting image plane (a line in 2D) to be interpolated having pixels shown as circles (top) and the corresponding locations of these pixels in the pre-deformed state, where the intensities of underlying regularly-spaced reconstructed voxels (which are not depicted here) are known a priori (bottom); (b) flagging image pixels close to element borders (marked with stars) in a 2D deformed mesh; and (c) flagging the projection (dashed-lines) of a $3 \mathrm{D}$ mesh on image pixels.

flagged with the corresponding element numbers as shown. Then, any pixel after a flagged one during a downwards traversal is guaranteed to be in that flagging element until another flagged pixel is encountered. The latter property (ii) is utilized for finding the pixels to flag near to mesh intersections. For instance in Fig. 1(b), simply rounding a line intersection to the next pixel location gives the pixel to flag without any further need for searching for this intersection in the image.

In 2D the flagging approach described above requires constant time per sliced element (any element that is intersected by the generated image). The sliced elements can be traversed easily using a pre-processed element neighborhood list. Consequently, the enclosing element in Step 1 above can be found in negligible time following this $\mathcal{O}(s)$ extra flagging processing per frame, where $s$ is the number of sliced elements. Note that finding an initial sliced element at every frame, which can be done by traversing the surface of the mesh, takes insignificant time.

\section{Extension to $3 D$ and the Proposed Algorithm}

In $3 \mathrm{D}$, the intersections of element edges with a planar image can be found using the deformed node positions. However, flagging only these intersections is not sufficient in 3D for marking the entire element borders. Instead, the projection of element surfaces must be found on the image. Let us demonstrate this on a tetrahedral mesh, where either 3 or 4 edges of an element may intersect a plane. The stars in Fig. 1(c) denote these edge intersections on the image plane. The surface projections depicted as dashed-lines are the boundaries where the enclosing element would change during a traversal of image pixels, thus these boundary pixels need to be flagged. For our 4-node linear tetrahedra, owing 
to the property (ii) above, we can employ Bresenham's line drawing algorithm to discretize and flag these projections on the image. In the example in Fig. 1(c), our preferred direction of traversal is top-down and hence only the top-halves of elements are flagged.

Note that more than one surface projection may be discretized on the same pixel, such as the pixel at the rightbottom corner of $e_{3}$ in Fig. 1(c). If $e_{5}$ were to be projected and flagged after $e_{3}$, this pixel would be flagged incorrectly. This could cause significant artifacts, since one mis-flagged pixel may result in a strip of mis-interpolated pixels below it due to top-down traversal. In order to avoid this, the elements are first topologically sorted using their top-down relationship prior to flagging their projections.

For given probe and mesh-node positions, the coordinate transformation matrix $T$ for undeforming an image pixel is constant for all the pixels in the same element. Therefore, it only needs to be computed once for a sliced element per frame. As a result, our proposed algorithm becomes:

1'. Form the sliced element list $L_{e}$ and their partial order by traversing the mesh along the image plane

2'. Topological-sort $L_{e}$

3'. Compute transformations for each element in $L_{e}$

4'. Flag top-half intersections of elements in $L_{e}$ on the image using Bresenham's line drawing algorithm

5'. For each image pixel $P$

5a'. If $P$ is flagged, switch to the new transformation $T$

$5 \mathrm{~b}$ '. Find the undeformed pixel position $T P$

$5 c$ '. Interpolate $T P$ in the given voxel data

\section{E. Computational Analysis}

Steps 1', 2', and 3' are $\mathcal{O}(s)$ operations, whereas Step 4' also depends on the image resolution. A further inspection reveals its order as $\mathcal{O}(\sqrt{n s})$ where $n$ is the number of image pixels. Recall that $s$ is the number of sliced elements, and therefore typically $s \ll n$. Note that the complexity of the entire algorithm is still the interpolation loop in Step 5, which runs in $\mathcal{O}(n)$. Nonetheless, the extra processing steps above, that are of lower computational order, save significant time during this loop's process, resulting in the anticipated speed gain. In particular, 5a' is a single memory access, 5b' consists of only 6 multiplications (from 2D image coordinates to 3D voxel data) and, assuming nearest-neighborhood interpolation, 5c' is a simple rounding operation.

\section{RESULTS}

For our experiments, a $60 \times 90 \times 90 \mathrm{~mm}$ tissue-mimicking gelatin phantom, having a soft cylindrical inclusion of $25 \mathrm{~mm}$ in diameter, was constructed. The phantom was meshed using off-the-shelf meshing software yielding 493 nodes and 1921 tetrahedra. The dimensions and the mesh of our phantom are depicted in Fig. 2(a)(top). The elasticity parameters for the FEM simulation were set to the approximate values known for the gelatin concentrations used. This phantom was imaged using a Sonix RP ultrasound machine from Ultrasonix Medical Corp. with a linear probe mounted on a precision motion stage. Vertical parallel slices with $1 \mathrm{~mm}$ separation

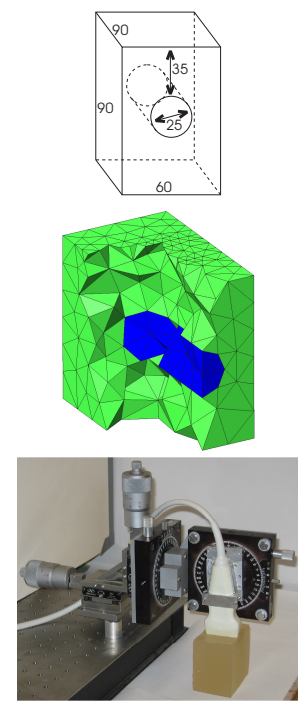

(a)
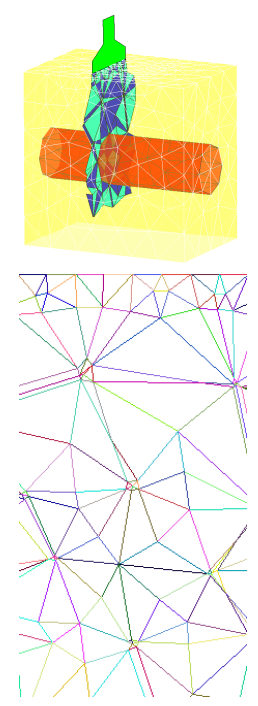

(b)



(c)
Fig. 2. (a) The phantom design, its mesh, and the image acquisition setup; (b) cross-sections of the elements sliced by the image and their boundaries discretized and flagged on the image prior to the interpolation; and (c) synthesized images of our tissue phantom with no deformation.

were acquired with care not to deform the phantom surface. The imaging setup is shown in Fig. 2(a)(bottom). The images are $37.5 \times 70 \mathrm{~mm}$ with a resolution of $220 \times 410$, which is the typical B-mode image resolution of this ultrasound machine. The voxel volume is considered to be the collection of these parallel slices. For this given image resolution, there is an average of 8750 voxels/element in our phantom mesh.

Fig. 2(b) shows the cross-sections of the traversed sliced elements of a sample case in 3D. It also demonstrates the corresponding pixels flagged on the image similarly to Fig. 1(c) by connecting and discretizing the edge intersections using Bresenham's algorithm. A sample image of the phantom with no deformation (probe pressure) is presented in Fig. 2(c). The deformation was applied in the form of the probe indenting the phantom vertically. It was simulated by fixing the bottom side of the phantom and applying the probe displacement to the mesh nodes on the top surface. The simulated images at 0,5 , and $10 \mathrm{~mm}$ surface displacements during two sample indentation configurations are presented in Fig. 3.

Our C++ implementation of the algorithm generates slices with the given resolution in less than $35 \mathrm{~ms}$ on a Pentium 4 $2.4 \mathrm{GHz}$ computer. Assessment of this elapsed time for various image sizes and resolutions showed that the number of pixels, $n$, determines the speed whereas the number of sliced elements, $s$, has little or no effect. This shows that the extra processing time introduced by our method in order to minimize the hidden cost during $\mathcal{O}(n)$ operation is justified.

The technique above has been implemented in a preliminary system for interactive ultrasound visualization with simulated deformation as seen in Fig. 4, where a SensAble Phantom ${ }^{\mathrm{TM}}$ Premium device mimics the probe. A visual interface displays the tissue and the probe in $3 \mathrm{D}$, as the ultrasound images are also synthesized by our algorithm and 


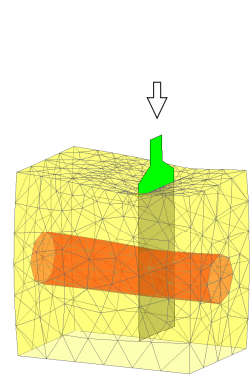

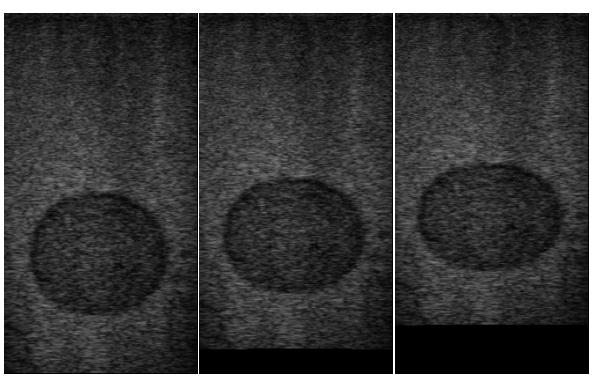

(a)

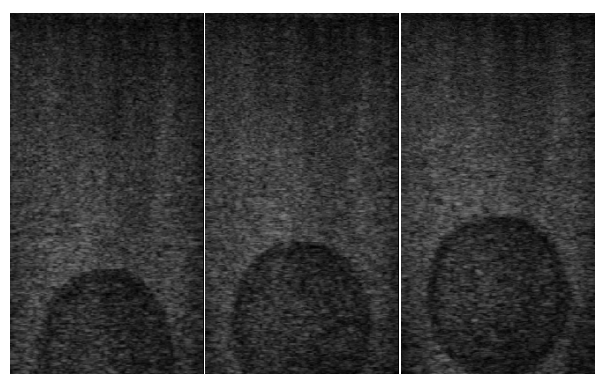

(b)

Fig. 3. Simulated images at 0,5 , and $10 \mathrm{~mm}$ indentation depths with vertical (a) and $45^{\circ}$-tilted (b) probe orientations (meshes are depicted for $10 \mathrm{~mm}$ only).

displayed in real-time. In this preliminary system, a simple contact handling method of applying the probe displacements onto the closest tissue node was chosen. During contact, a feedback force normal to the tissue surface and modeled by a spring is applied on user's hand.

\section{DISCUSSION}

A visual inspection of the images simulating probe pressure shows them to be adequate for medical simulators. In order to further evaluate these images, the realism of the synthesis such as realistic speckle patterns should be assessed. An attempt to directly compare the simulated images to acquired ones may fail since it mainly depends on the deformation model, not the interpolation technique.

For deformation, we employed the FEM with 4-node linear tetrahedra due to its shape function being simple, continuous, and easily invertible. Nonetheless, the presented method also applies to element geometries other than tetrahedra. However, note that planar projections of non-linear elements are not necessarily lines, and therefore the simple Bresenham's algorithm cannot be used to flag projections. Care must be taken not to compromise the continuity of shape functions at element borders, since this will cause artifacts.

Note that increasing the object dimensions while keeping the element sizes the same has little or no effect on our algorithm. Although the number of mesh nodes/elements may directly affect the performance of the deformation model, it only has a minor contribution to the complexity of our synthesis approach by changing the number of sliced elements. Even then, a quick analysis shows that this is negligible compared to the dominant effect of the image resolution on the computation time.

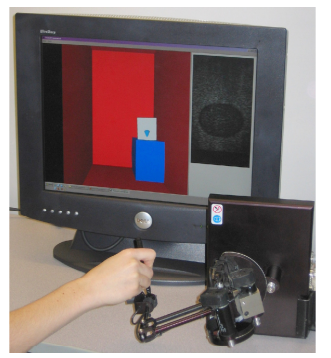

Fig. 4. Our real-time ultrasound scanning simulator.

\section{CONCLUSIONS AND FUTURE WORK}

In this paper, a fast technique for synthesizing planar images in deformed meshes of tissue models, the 3D image voxel data of which are given a priori in their predeformed configuration, was presented. A pixel flagging technique that enables fast identification of mesh elements enclosing each image pixel during deformations given by mesh node displacements was developed. This allows us to simulate medical images of considerable sizes at visual frame rates, that are suitable for real-time applications. This technique was implemented to simulate the ultrasound scanning of a deformable phantom. The results show that the proposed method produces realistic-looking B-mode images. Next, the synthesized deformed images will be quantitatively compared with acquired ones. Later, this image synthesis technique will be integrated with a haptic brachytherapy simulator using FEM-based needle insertion models and the synthesized transrectal ultrasound images will be assessed by experts.

\section{REFERENCES}

[1] J. F. Krücker, G. L. LeCarpentier, J. B. Fowlkes, and P. L. Carson, "Rapid elastic image registration for 3-D ultrasound," IEEE Trans Med Imag, vol. 21, no. 11, pp. 1384-1394, 2002.

[2] D. Zikic, W. Wein, A. Khamene, D.-A. Clevert, and N. Navab, "Fast deformable registration of 3D-ultrasound data using a variational approach," in MICCAI, Copenhagen, Denmark, 2006, pp. 915-923.

[3] R. N. Rohling, A. H. Gee, and L. Berman, "A comparison of freehand three-dimensional ultrasound reconstruction techniques," Med Image Anal, vol. 3, no. 4, pp. 339-359, 1999.

[4] A. Gee, R. Prager, G. Treece, C. Cash, and L. Berman, "Processing and visualizing three-dimensional ultrasound data," British J Radiology, vol. 77, pp. S186-S193, 2004.

[5] J. A. Jensen, "A model for the propagation and scattering of ultrasound in tissue," J Acoust Soc Am, vol. 89, pp. 182-191, 1991.

[6] D. Aiger and D. Cohen-Or, "Real-time ultrasound imaging simulation," Real-Time Imaging, vol. 4, no. 4, pp. 263-274, 1998.

[7] H. Maul, A. Scharf, P. Baier, M. Wüstemann, H. H. Günter, G. Gebauer, and C. Sohn, "Ultrasound simulators: Experience with sonotrainer and comperative review of other training systems," Ultrasound Obstet Gynecol, vol. 24, pp. 581-585, 2004.

[8] D. Henry, J. Troccaz, J. L. Bosson, and O. Pichot, "Ultrasound imaging simulation: Application to the diagnosis of deep venous thromboses of lower limbs," in MICCAI, 1998, pp. 1032-1040.

[9] D. d'Aulignac, C. Laugier, J. Troccaz, and S. Vieira, "Towards a realistic echographic simulator," Med Image Anal, vol. 10, pp. 71$81,2005$.

[10] C. B. Barber, D. P. Dobkin, and H. T. Huhdanpaa, "The Quickhull algorithm for convex hulls," ACM Trans Math Softw, vol. 22, no. 4, pp. 469-483, 1996. 